\title{
CLASIFICACIÓN DE SUELOS BASADA EN EL CÁLCULO DE RAZONES ESPECTRALES EN SITIOS DONDE SE UBICAN ESTACIONES ACELEROGRÁFICAS DE AMÉRICA CENTRAL. CASOS DE EL SALVADOR, NICARAGUA Y COSTA RICA
}

\author{
SOIL CLASSIFICATION BASED ON SPECTRAL RATIOS WHERE CENTRAL \\ AMERICAN ACCELEROGRAPHIC STATIONS ARE LOCATED. CASES OF EL \\ SALVADOR, NICARAGUA AND COSTA RICA
}

\author{
Víctor Schmidt \\ Laboratorio de Ingeniería Sísmica, Inst. de Investigaciones en Ingeniería, \\ Facultad de Ingeniería, Universidad de Costa Rica. \\ 2060 San Pedro de Montes de Oca, San José, Costa Rica \\ victor.schmidt@ucr.ac.cr
}

(Recibido: 25/08/2010; aceptado: 01/06/2011)

\begin{abstract}
This research applies a common soil classification for sites where most of the Central American accelerographic stations are located. It is very necessary to adopt a uniform classification procedure for all sites, because usually the information provided by local agencies (accelerographic network administrators) is incomplete or based on subjective interpretations read from maps or observation of the surface geology. Moreover, precise knowledge of the soil type in which accelerograms are recorded is essential to validate the records for future researches. It was considered the information provided by each local agency to assign the soil type for each site, as well as the USGS (U.S. Geological Survey) Vs30 map and it was followed the procedure proposed by Zhao et al. (2006) based on the calculation of spectral average ratios of the horizontal component on the vertical, obtained from all available records at each station. Finally, it is assigned a score to each site according to coincidence or not of different mentioned classification criteria. 134 station sites were studied, which received the following qualification: A (26\%), B (32\%), C (17\%) and D (25\%), being A the best category and D the worst one. Only few stations were classified as S I (rock) and S IV (soft soil). Comparing the spectral ratios obtained by other authors for Japan, the amplifications obtained in the present study tend to be lower in almost the whole range of periods for the four considered soil types.
\end{abstract}

Keywords: Soil types, amplificaction, accelerograms, Central America.

RESUMEN: En esta investigación se aplica un método de clasificación de suelos común para los sitios donde se ubican la mayoría de las estaciones acelerográficas de América Central. Se ha evidenciado la necesidad de aplicar un procedimiento de clasificación homogéneo para todos los emplazamientos, debido a que generalmente la información 
que aportan las agencias locales (administradores de las redes acelerográficas), es incompleta o subjetiva, basada en interpretaciones de mapas o solamente en la observación de la geología superficial. Por otro lado, el conocimiento preciso del tipo de suelo en el cuál se registran los acelerogramas es fundamental para el uso de esos registros en el desarrollo de investigaciones. Para la asignación del tipo de suelo se consideró la información aportada por cada agencia local, así como mapas de Vs30 propuestos por el USGS (US Geologiva Survey) y se sigue el procedimiento propuesto por Zhao et al. (2006), que se basa en el cálculo del promedio de las razones espectrales de la componente horizontal sobre la vertical, obtenidas a partir de todos los registros disponibles en cada estación. Finalmente, se le asigna una calificación a cada sitio según la coincidencia o no de los distintos criterios de clasificación considerados. Se estudiaron 134 emplazamientos de estaciones acelerográficas, que obtuvieron las siguientes calificaciones: A (26\%), B (32\%), C (17\%) y D (25\%), siendo A la mejor categoría y D la más deficiente. Se obtuvieron pocas estaciones clasificadas como S I (roca) y S IV (suelo blando). Al comparar las razones espectrales con las obtenidas para Japón por otros autores, las del presente estudio tienden a ser menores en casi todo el rango de periodos para los cuatro tipos de suelo considerados. Palabras clave: Tipos de suelo, amplificación, acelerogramas, América Central.

\section{INTRODUCCIÓN}

Es responsabilidad de toda red acelerográfica proporcionar no solo los acelerogramas obtenidos al ocurrir un movimiento sísmico fuerte, sino también las condiciones del suelo en las que estos fueron registrados, debido a que los daños observados en la superficie suelen estar estrechamente relacionados con las características del sitio.

Existen ciertos tipos de suelo capaces de modificar las ondas sísmicas, tanto en la duración como en la amplitud y en el contenido frecuencial, lo que es conocido como efectos de sitio o respuesta del suelo. Debido a esto, es fundamental para el investigador conocer el tipo de emplazamiento asociado a cada registro, de modo que pueda tomar en cuenta o bien, descartar la presencia de este fenómeno en los acelerogramas con los que vaya a desarrollar su investigación.

Específicamente en lo referente a las ecuaciones predictivas del movimiento del suelo o ecuaciones de atenuación, una de las variables más importantes es la que representa la respuesta del suelo, ya que de ella dependen en gran medida las amplitudes estimadas con base en dichos modelos.

Se ha observado que cuando existe de una incorrecta clasificación de suelos, se producen grandes variaciones en los niveles estimados de la amenaza sísmica, principalmente cuando se utilizan ecuaciones de atenuación obtenidas a partir de registros que provienen de sitios con clasificaciones del suelo poco confiables (Zhao et al., 2006). Por lo tanto, para lograr una adecuada participación del factor que determina la respuesta del suelo en los estudios de atenuación de las ondas sísmicas, es fundamental contar con una clasificación confiable de los tipos de emplazamiento donde se ubican las estaciones acelerográficas que se tomarán en cuenta en el análisis, lo que permitirá obtener modelos más robustos y confiables.

En forma semejante, si un investigador pretende proponer espectros de diseño de estructuras a partir de registros acelerográficos reales, es fundamental conocer las condiciones del suelo donde se obtuvieron los acelerogramas, ya que este tipo de espectros dependen de las condiciones locales de cada sitio.

A modo de antecedente, las redes acelerográficas de América Central cuentan con una clasificación de suelos propia (Bundschuh \& Alvarado, 2007) pero esta resulta ser subjetiva y difícilmente homogénea, ya que se basa en interpretaciones de mapas geológicos y geotécnicos hechas por distintas personas, o bien, en pruebas que se realizaron en algunos sitios sin seguir un procedimiento de clasificación estándar. Además, no existe ningún estudio debidamente publicado donde se haga referencia a una clasificación de suelos para estaciones acelerográficas propiamente dicha.

De acuerdo con las normas sísmicas vigentes y considerando el hecho de que los códigos en países americanos generalmente toman como 
base la normativa de Estados Unidos, el objetivo principal del presente estudio es el de clasificar los suelos donde se ubican las estaciones acelerográficas con mayor número de registros existentes en El Salvador, Nicaragua y Costa Rica, tomando como base la norma NEHRP (Nacional Earthquake Hazards Reduction Program, BSSC, 2003), que suele ser un referente importante a nivel mundial.

NEHRP define seis tipos distintos de suelo que van desde la categoría A hasta la F, siendo los más comunes los mostrados en el cuadro 1 .

Donde Vs30 es una clase de promedio de velocidades de ondas de corte correspondiente a los primeros $30 \mathrm{~m}$ medidos desde la superficie. $\mathrm{Su}$ expresión matemática es:

$$
V s 30=\frac{\sum_{i=1}^{N} d_{i}}{\sum_{i=1}^{N} \frac{d_{i}}{V_{s i}}}
$$

Siendo di el espesor de cada capa de suelo del perfil hasta alcanzar los $30 \mathrm{~m}$ de profundidad, $V_{s i}$ la velocidad de onda cortante de cada capa $i$ en $\mathrm{m} / \mathrm{s}$ y $\mathrm{N}$ el número de capas hasta alcanzar los $30 \mathrm{~m}$.

El término NSPT representa la resistencia a la penetración estándar y SU el esfuerzo cortante en condición no drenada, ambos obtenidos como promedio para las capas de suelo consideradas.
De acuerdo con esta norma, para lograr una adecuada clasificación de suelos es necesario hacer una estimación de los términos: Vs30, $\mathrm{N}_{S P T}$ y $\mathrm{S}_{U}$. Sin embargo, para su obtención se requiere llevar a cabo una serie de pruebas geotécnicas y geofísicas que generalmente demandan grandes inversiones, especialmente económicas y de tiempo.

Debido a lo anterior, se ha investigado sobre métodos que permitan aplicar criterios de clasificación que sean económicos y sistemáticos, que posibiliten una adecuada determinación del tipo de suelo de los sitios donde no se cuenta con pruebas geofísicas.

Para esta investigación se seleccionó un método de clasificación basado principalmente en el cálculo de razones espectrales obtenidas a partir de acelerogramas registrados en cada estación donde se cuente con una suficiente cantidad de registros, lo que permitirá asignarle a cada sitio un tipo de suelo. Estos resultados tienen un carácter preliminar y deberán ser comparados con los obtenidos con pruebas geofísicas detalladas que se lleven a cabo en los lugares bajo estudio en el futuro.

Una vez que se logre la correcta clasificación de los suelos donde se ubican las estaciones acelerográficas, se obtendrán impactos positivos en diversas áreas de investigación como:

- Modelos de atenuación de la onda sísmica más precisos: se podrán obtener factores de amplificación en función de la frecuencia, para cada tipo de suelo B, C, D o E respecto a la roca. Esto

Cuadro 1

Tipos de suelo definidos por la NEHRP según sus características geotécnicas y geofísicas más importantes (BSSC, 2003). No se describe el tipo F por presentar características poco comunes y que no dependen de Vs30

\begin{tabular}{cc}
\hline Clase & Características \\
\hline A & $\begin{array}{c}\text { Roca dura con Vs } 30>1500 \mathrm{~m} / \mathrm{s} \\
\text { Roca con } 760 \mathrm{~m} / \mathrm{s}<\mathrm{Vs} 30 \leq 1500 \mathrm{~m} / \mathrm{s}\end{array}$ \\
C & $\begin{array}{c}\text { Suelo muy denso y roca suave con } 360<\mathrm{Vs} 30 \leq 760 \text { ó NSPT } \\
>50 \text { o SU }>100 \mathrm{KPa}\end{array}$ \\
D & Suelo firme con $180 \mathrm{~m} / \mathrm{s} \leq \mathrm{Vs} 30 \leq 360 \mathrm{~m} / \mathrm{s}$ ó $15 \leq \mathrm{NSPT} \leq 50$ \\
ó $50 \mathrm{Kpa} \leq \mathrm{SU} \leq 100 \mathrm{KPa}$
\end{tabular}


permitirá realizar estudios de amenaza sísmica en los que los efectos de sitio sean debidamente tomados en cuenta y así hacer una estimación más precisa de las solicitaciones sísmicas que permitan lograr un diseño sismorresistente de estructuras más eficiente.

- Registros acelerográficos: pueden ser comparados y complementados con otros provenientes de otras partes del mundo en condiciones de suelo semejantes, de manera que se puedan llevar a cabo estudios de efectos de sitio en zonas donde los registros acelerográficos sean escasos $\mathrm{o}$ inexistentes.

- Espectros de diseño: los espectros de respuesta se podrán agrupar por tipo de suelo y al aplicar procedimientos adecuados, será posible proponer nuevos espectros que puedan ser incorporados en normas de diseño.

- Luego de la ocurrencia de un sismo, será posible establecer la correlación de los daños observados en un sitio específico con el movimiento de suelo registrado, por medio de algún parámetro de correlación obtenido del acelerograma. Esto solamente será posible si el tipo de suelo está debidamente identificado.

\section{MÉTODO SELECCIONADO Y METODOLOGÍA PROPUESTA}

El método seleccionado para la clasificación de suelos se basa la propuesta hecha por Zhao et al. (2006). Este les permitió a los autores crear un esquema de clasificación de suelos para Japón, a partir de todos los registros sísmicos que ellos disponían para cada estación. También contaron con información proveniente de perfiles geotécnicos construidos con base en pruebas geofísicas (boreholes) en esos mismos sitios, hasta profundidades en las que las Vs alcanzan los 600 a $700 \mathrm{~m} / \mathrm{s}$, lo que les permitió corroborar el método propuesto.

Ellos basan su propuesta en la estimación de la razón espectral $\mathrm{H} / \mathrm{V}$ a partir del cálculo de los espectros de respuesta para el 5\% de amortiguamiento para las componentes horizontales $(\mathrm{H}) \mathrm{y}$ para la vertical (V) y se ajustaron a la clasificación de suelos existente en Japón. Evidenciaron además que el uso de promedios entre todas las
$\mathrm{H} / \mathrm{V}$ obtenidas en cada estación eliminan picos extremos o anomalías en ciertas razones específicas, por lo que en general cada sitio mantenía un comportamiento estable (formas semejantes en las $\mathrm{H} / \mathrm{V}$ de un mismo sitio). Observaron además que estos promedios no se veían fuertemente afectados si los datos se separaban por distancia hipocentral, magnitud o profundidad al hipocentro.

El método propuesto por Zhao et al. (2006), no puede ser fácilmente justificado con modelos teóricos; por esta razón se le considera un procedimiento empírico.

La metodología propuesta en la presente investigación consiste en cinco pasos principales:

1- Selección de las estaciones acelerográficas de El Salvador, Nicaragua y Costa Rica que serán clasificadas. Esto se hace en función del número y la calidad de los registros acelerográficos disponibles. Cálculo de las razones espectrales H/V.

2- Clasificación del sitio a partir del valor del pico máximo identificado en el promedio de las razones espectrales y comparación con los rangos para $T_{0}$ presentados en el cuadro 2. Esto será especialmente útil para la identificación del clases SC III y IV, siempre y cuando el número de registros disponible sea de al menos 5 (Zhao et al., 2006).

3- Estimación el índice propuesto por Zhao et al. (2006) pero con datos de América Central:

$$
S I=\frac{2}{N} \sum_{i=1}^{N} F\left(-a b s\left[\ln \left(\mu_{i}\right)-\ln \left(\bar{\mu}_{K i}\right)\right]\right)
$$

Donde:

$\mathrm{K}$ : número de clase de sitio, desde S I hasta S IV

$\mathrm{N}$ : número total de periodos, que en este caso se usarán 20

$\mathrm{F}($ ): función de distribución normal acumulativa

$\bar{\mu}_{\mathrm{i}}$ : valor $\mathrm{H} / \mathrm{V}$ de amplitud promedio para el sitio de interés, para el periodo $i$,

$\mu_{\mathrm{Ki}}:$ valor promedio de amplitud de $\mathrm{H} / \mathrm{V}$ para todos los sitios clase $\mathrm{K}$, obtenido del promedio entre todos los sitios de la base de datos para el periodo i. Al no contarse con estos promedios para América Central, se trabajará con los obtenidos para Japón, ya que corresponden a una gran cantidad de sitios 
Cuadro 2

Clasificación usada en Japón para prácticas de diseño en ingeniería y utilizada por Zhao et al. (2006), para la clasificación de los sitios a partir de los picos promedio (periodos predominantes) observados en las razones $\mathrm{H} / \mathrm{V}$

\begin{tabular}{cccc}
\hline Clases de sitio & $\begin{array}{c}\text { Periodo natural del sitio } \\
\mathrm{T}_{0}(\mathrm{~s})\end{array}$ & $\begin{array}{c}\text { Promedio de las velocidades } \\
\text { de onda cortante }(\mathrm{m} / \mathrm{s})\end{array}$ & $\begin{array}{c}\text { Equivalencia con la clasifi- } \\
\text { cación del NEHRP }\end{array}$ \\
\hline S I (roca/suelo rígido) & $\mathrm{T}_{0}<0,2$ & $\mathrm{Vs} 30>600$ & $\mathrm{~A}+\mathrm{B}$ \\
S II (suelo firme) & $0,2=\mathrm{T}_{0}<0,4$ & $300<\mathrm{Vs} 30=600$ & $\mathrm{C}$ \\
S III (suelo medio) & $0,4=\mathrm{T}_{0}<0,6$ & $200<\mathrm{Vs} 30=300$ & $\mathrm{D}$ \\
S IV (suelo blando) & $\mathrm{T}_{0}=0,6$ & $\mathrm{Vs} 30=200$ & $\mathrm{E}$ \\
\hline
\end{tabular}

bien documentados y estimados a partir de muchos registros, lo que los hace muy confiables y estables.

El índice SI es igual a la unidad si el promedio de las razones espectrales para un sitio en particular es igual al promedio de todos los de su categoría; en caso contrario es menor que la unidad. Para una estación específica, el SI es calculado para cada clase K y este sitio será clasificado en la clase cuyo valor de SI sea el mayor.

Al usar razones espectrales a partir de espectros de respuesta, tal y como es el caso que se propone, el cálculo para el 5\% de amortiguamiento tiene un efecto de suavizado que es semejante para todos los registros (no requiere la aplicación de suavizados específicos para cada registro). Por otro lado, en este caso no es necesario identificar el arribo de las ondas $\mathrm{S}$, como sí es requisito cuando se trabaja con espectros de Fourier, ya que es a partir del arribo de estas ondas cuando se observan los efectos de sitio y esta identificación implica una gran inversión de tiempo para lograr hacerlo en cada uno de los registros disponibles. Al usarse espectros de respuesta, como las aceleraciones espectrales máximas suelen ocurrir en un tiempo muy cercano al arribo de las ondas $\mathrm{S}$, generalmente quedan contenidas en el espectro de respuesta, tanto en la componente horizontal como en la vertical.

4- Revisión la información proporcionada por cada país (descripciones geológicas hechas localmente), que están resumidos en Bundschuh \& Alvarado, 2007. En los casos donde no se contó con este criterio de clasificación, se calculó la Vs30 con base en los mapas generados siguiendo el procedimiento del USGS (USGS Earthquake Hazards Program, Custom Vs30 Mapping,
Estimates of site conditions from topographic slope, http://earthquake.usgs.gov/hazards/apps/ vs30/custom.php). Consiste en seleccionar un área geográfica dentro de la página web citada y leer directamente los valores de Vs30 que han sido correlacionados por los autores con las pendientes de la topografía de la zona. En términos generales, a las mayores pendientes se les asocia valores de Vs30 altos (suelos más antiguos) y a pendientes menores o zonas más planas, Vs30 bajas (suelos más recientes).

5- Calificación de cada sitio basándose en la coincidencia o no de los métodos propuestos en los puntos 2, 3 y 4 de la metodología propuesta. A continuación se describen los tres criterios considerados:

Criterio 1: tipo de suelo reportado por la institución local, revisado con base en la geología local y con la Vs30 obtenida de los mapas generados a partir del USGS.

Criterio 2: tipo de suelo con base en el periodo fundamental obtenido a partir de las razones espectrales.

Criterio 3: tipo de suelo con base en el cálculo del índice de clasificación SIK.

Así pues, la escala de calificación aplicada a todos los sitios define cuatro posibilidades, de la A a la $\mathrm{D}$, siendo la $\mathrm{A}$ la mejor:

A: coincidencia entre los tres criterios.

B: coincidencia entre el criterio 1 y el 2 o del 1 y el 3 .

C: coincidencia entre los criterios 2 y 3

D: no coincidencia entre los criterios o pocos registros en el sitio.

Se considera que el caso $\mathrm{B}$ es más confiable que $\mathrm{C}$, debido a que el $\mathrm{B}$ proviene de la comparación de criterios diferentes, basados en 
información totalmente distinta ya que el criterio 1 se basa en observación de la geología superficial o mapas, mientras que el 2 y el 3 corresponden a la aplicación de métodos empíricos, lo que hace que la coincidencia entre $1 \mathrm{y}$ 2 o 1 y 3 genere más confianza al clasificar ese sito que el caso $\mathrm{C}$, que está totalmente basado en métodos empíricos y de alguna manera, correlacionados.

La calificación D significa que no es confiable del todo la asignación del tipo de suelo al seguir esta metodología.

\section{RESULTADOS}

Los suelos predominantes en América Central son muy variados, como consecuencia de la complejidad del medio ambiente en el que interactúan diferentes tipos de roca, el clima, la topografía irregular y de los organismos vivos, incluidos los seres humanos. En las montañas y valles a lo largo del eje central de Centroamérica predominan rocas volcánicas, incluyendo los flujos de lava y depósitos de cenizas, así como rocas piroclásticas, con excepción de un intrusivo situado en el sureste de Costa Rica. Las zonas de montaña se encuentran hacia la costa del Pacífico (sistemas montañosos generados por los procesos de subducción). Por otro lado, las llanuras costeras del Caribe dominan grandes extensiones. En los cauces de los ríos predominan los procesos de erosión y al estar deforestados en muchos casos, se agrava esta situación (Centro Nacional de Registros de El Salvador, 2000; Ineter \& BRG, 2004; Denyer \& Alvarado, 2007).

La fig. 1 muestra un mapa simplificado de las condiciones geológicas superficiales que prevalecen en América Central, basado en Bundschuh \& Alvarado (2007). Se observa el predominio de rocas volcánicas y también de rocas sedimentarias en los sitios donde se encuentra ubicada la mayor parte de las estaciones acelerográficas de El Salvador, Nicaragua y Costa Rica. También se puede deducir que pocas estaciones se ubican sobre rocas competentes.

Se llevó a cabo la clasificación de los suelos de 134 estaciones, que están repartidas de la siguiente forma: 26 en Nicaragua, 43 en Costa Rica y 65 El Salvador.

Debido a la gran cantidad de razones espectrales generadas, se han seleccionado a modo de ilustración algunos casos que se consideran representativos de los principales resultados obtenidos. Estos son mostrados en las figs. 2, 3 y 4.

La fig. 2a muestra los resultados para la estación AALJ (Costa Rica), en la que hay una clara identificación de $\mathrm{T}_{0}$ ya que existe una buena cantidad de registros disponibles, lo que permite obtener una razón espectral promedio bien definida y una clara correspondencia entre el periodo fundamental y el tipo de suelo S IV (blando).

Algo semejante sucede con la estación NCHI (Nicaragua, fig. 2b), pero en este caso la clasificación obtenida corresponde a suelo tipo S III (medio).

Las figuras 3a (ESTE, El Salvador) y $3 b$ (NJIN, Nicaragua) muestran resultados para condiciones de suelo S I (roca) y S II (suelo firme), respectivamente. En ambos casos, los picos de máxima amplitud de las razones espectrales promedio son bien definidos, pero en el caso de NJIN se cuenta con un menor número de registros respecto a los demás casos ejemplificados.

Las figs. 4a y $4 \mathrm{~b}$ muestran casos en los que resulta difícil obtener una clasificación del sitio debido a las irregularidades en las formas de las razones espectrales promedio. En la 4a (estación SFRA, Costa Rica) se observa una H/V muy plana y dos picos sobresalientes, por lo que resulta difícil identificar el $\mathrm{T}_{0}$ correspondiente a ese sitio, mientras que en la fig. 4.3b (NGRA, Nicaragua) se muestra un caso que se presentó varias veces, donde la forma de la razón espectral promedio no sigue una tendencia definida, debido probablemente a la poca cantidad de registros disponibles.

Los cuadros 3, 4 y 5 muestran los resultados finales de la clasificación, donde se indica para cada sitio: el país, el código de la estación, el criterio de clasificación de acuerdo con la geología local, el número de componentes (dos componentes horizontales por cada registro), los resultados de la clasificación usando el método de identificación del período fundamental $\left(\mathrm{T}_{0}\right)$, los índices calculados según Zhao et al. (2006), la 


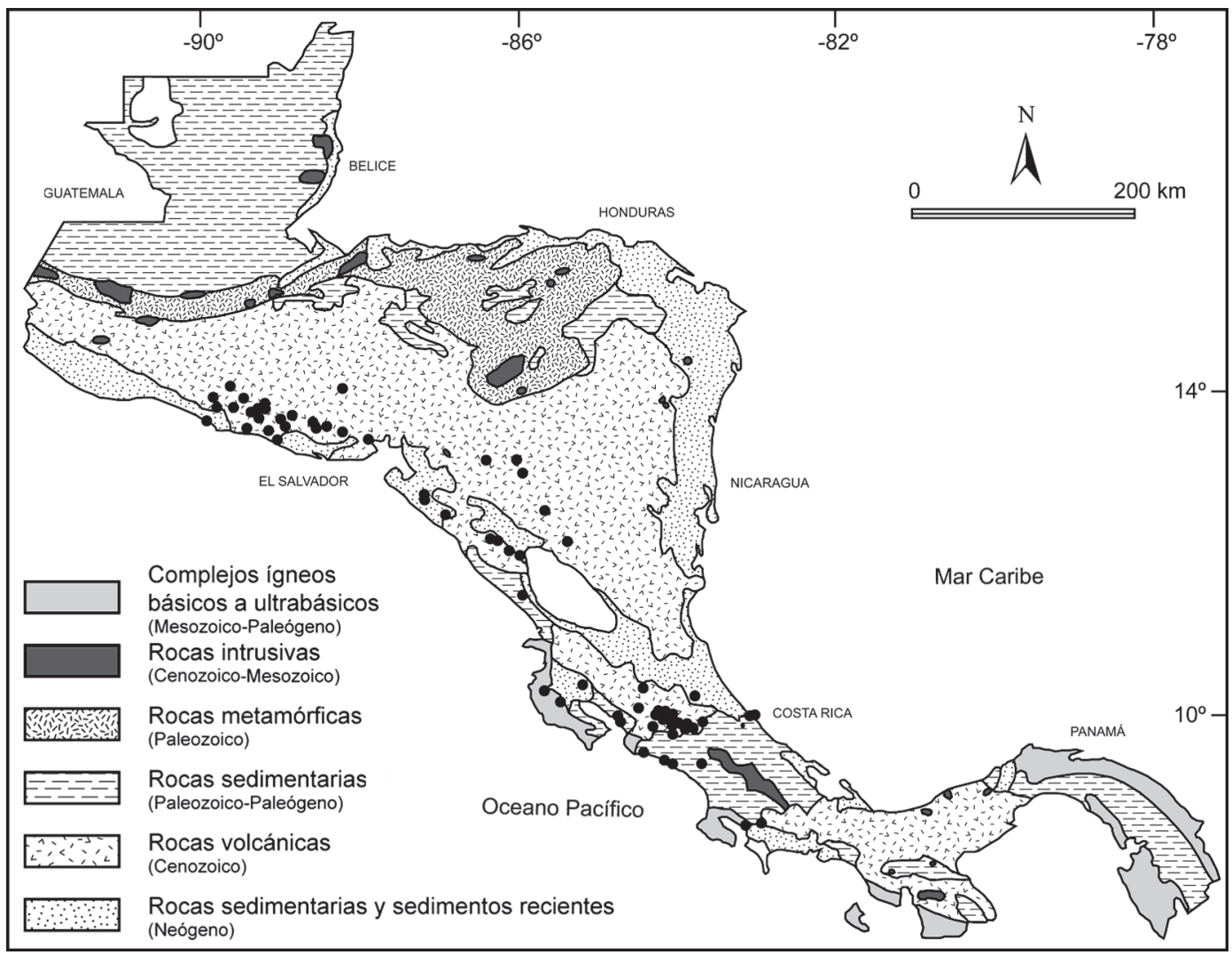

Fig. 1: Mapa geológico simplificado de América Central basado Bundschuh \& Alvarado (2007) y ubicación de las principales estaciones acelerográficas de El Salvador, Nicaragua y Costa Rica (puntos negros). Mapa digitalizado por César Sequeira, LIS.

clasificación final usando los distintos criterios y la calificación asignada.

El cuadro 3 corresponde al caso de Nicaragua (26 estaciones), donde se obtuvo que un $38 \%$ de las mismas logró una calificación A (coincidencia entre los tres criterios de clasificación), un $27 \%$ califican con $\mathrm{B}$, un $12 \%$ con $\mathrm{C}$ y un $23 \%$ con $\mathrm{D}$.

Considerando que las calificaciones A y B son las mejores, ya que corresponden a la coincidencia de al menos dos métodos de clasificación no correlacionados, un $65 \%$ de las estaciones de Nicaragua fueron bien calificadas. Sin embargo, en ese país se observan algunos casos particulares como la estación NINE, en la que hay 25 registros (50 componentes horizontales), pero no hay coincidencia entre los criterios de clasificación y el gráfico de las razones espectrales es bastante irregular, sin una tendencia que permita identificar $\mathrm{T}_{0}$, además de diferencias muy significativas entre las amplitudes de razones espectrales analizadas individualmente. Se presentan otros casos especiales como NMAI y NMAT donde las razones espectrales promedio son bastante planas, lo que impide reconocer el periodo fundamental y dificulta aplicar el método de clasificación basado en los índices, debido a que estos tienen valores muy semejantes para los cuatro tipos de suelo considerados. Sin embargo, estos casos corresponden a estaciones con pocos registros.

Para Nicaragua, la mayor parte de las estaciones clasificadas resultaron ser S I (roca), pero muchas de ellas tienen clasificación $\mathrm{D}$, por lo que no se puede asegurar que realmente haya una buena representación de este tipo de emplazamiento dentro de todo el conjunto de datos que aporta este país.

Costa Rica aporta 43 estaciones al estudio (cuadro 4), de las cuales un 37\% recibieron calificación 

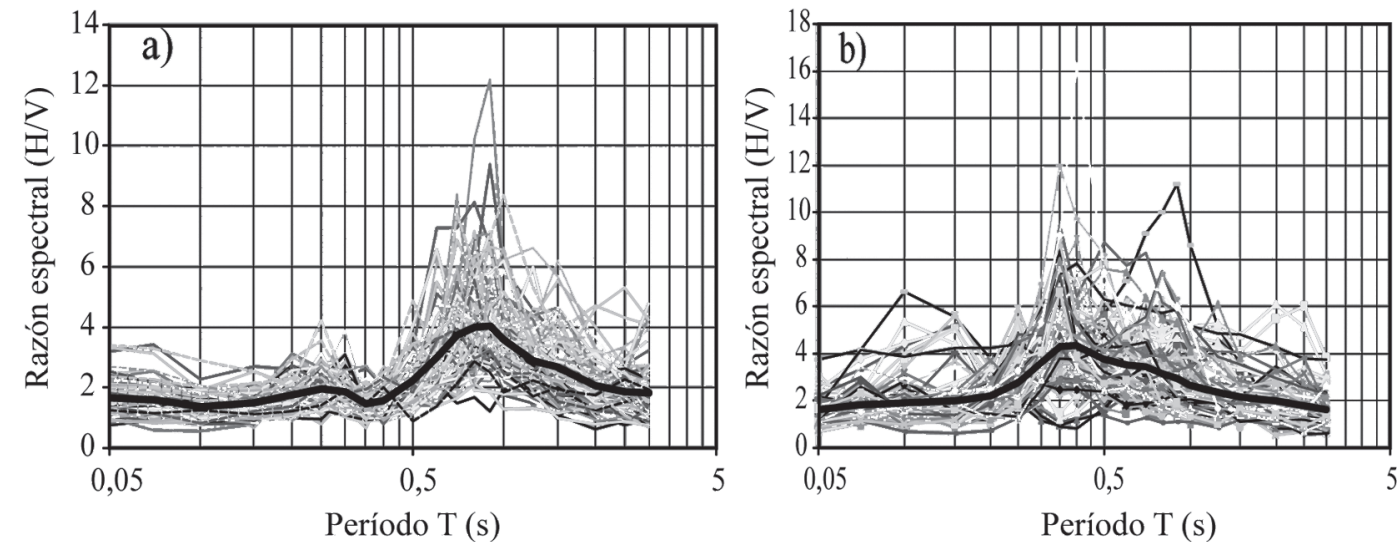

Fig. 2: Gráficos de razones espectrales para suelos: a) muy blando (estación AALJ, Costa Rica y b) blando (estación NCHI, Nicaragua). Los promedios son resaltados con línea negra más gruesa.

A y un 33\% fueron calificadas como B, lo que implica que un $70 \%$ del total de las estaciones cumple con al menos la coincidencia de dos criterios de clasificación. Por otro lado, las calificaciones C y D suman un $30 \%$ del total para este país, lo que implica que ese porcentaje no cumple satisfactoriamente con los criterios de calificación propuestos.

Los niveles de amplitud para $T_{0}$ observados en las razones espectrales son muy variables, entre 2,18 y 5,4 y no siempre concordantes con los niveles promedio que para cada tipo de suelo que obtuvieron Zhao et al. (2006) según fig. 7a, aunque sí se ubican dentro de los mismos rangos que esos autores proponen.

Para Costa Rica se observan pocas estaciones clasificadas como roca (solamente un $7 \%$ )

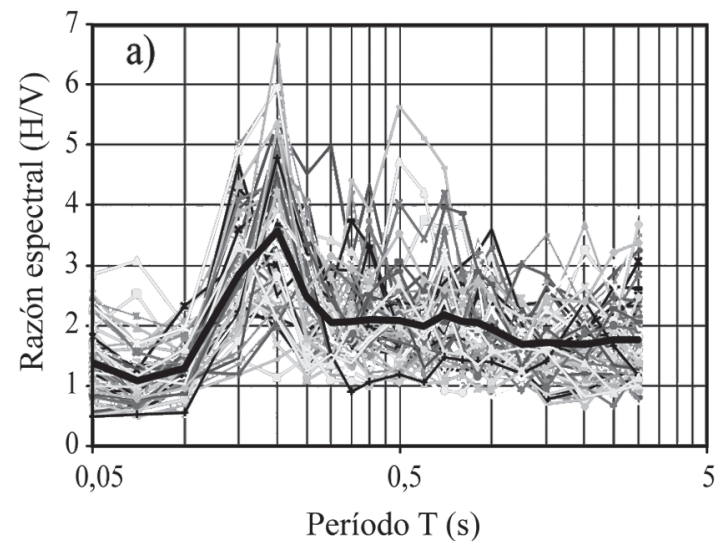

correspondiendo la mayoría a suelo tipo S II y S III. Esto es concordante con el Mapa Geolgógico de Costa Rica 2007 (Denyer \& Alvarado, 2007) y con Bundschuh \& Alvarado (2007), fig. 1, donde claramente los afloramientos de rocas no son predominantes ni en el territorio costarricense ni en el centroamericano en general, sobretodo en zonas pobladas donde se ubican la mayor parte de las estaciones acelerográficas.

La mayor parte de las estaciones que aportan más cantidad de registros de Costa Rica fueron calificadas en categorías A y B, lo que asegura la confiabilidad de su uso en futuras investigaciones.

En el caso de El Salvador (cuadro 5), se clasificaron 65 estaciones de las cuales solamente un $14 \%$ obtuvo calificación A, un 39\% se calificó como

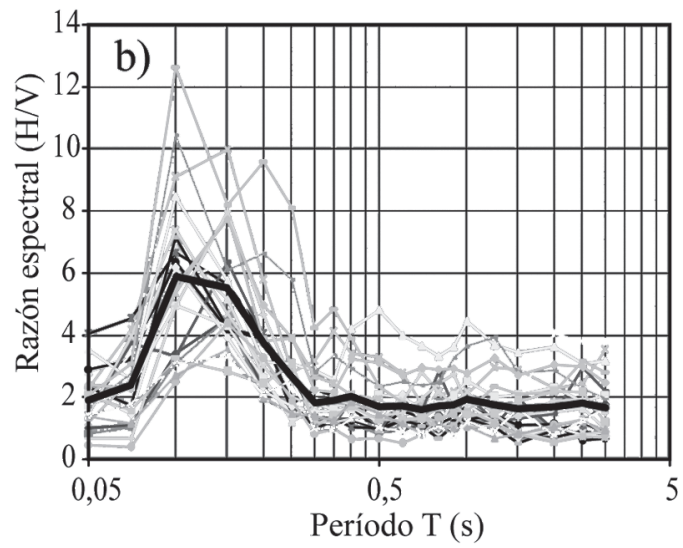

Fig. 3: Gráficos de razones espectrales para suelos: a) roca (estación ESTE, El Salvador y b) firme (estación NJIN, Nicaragua). 

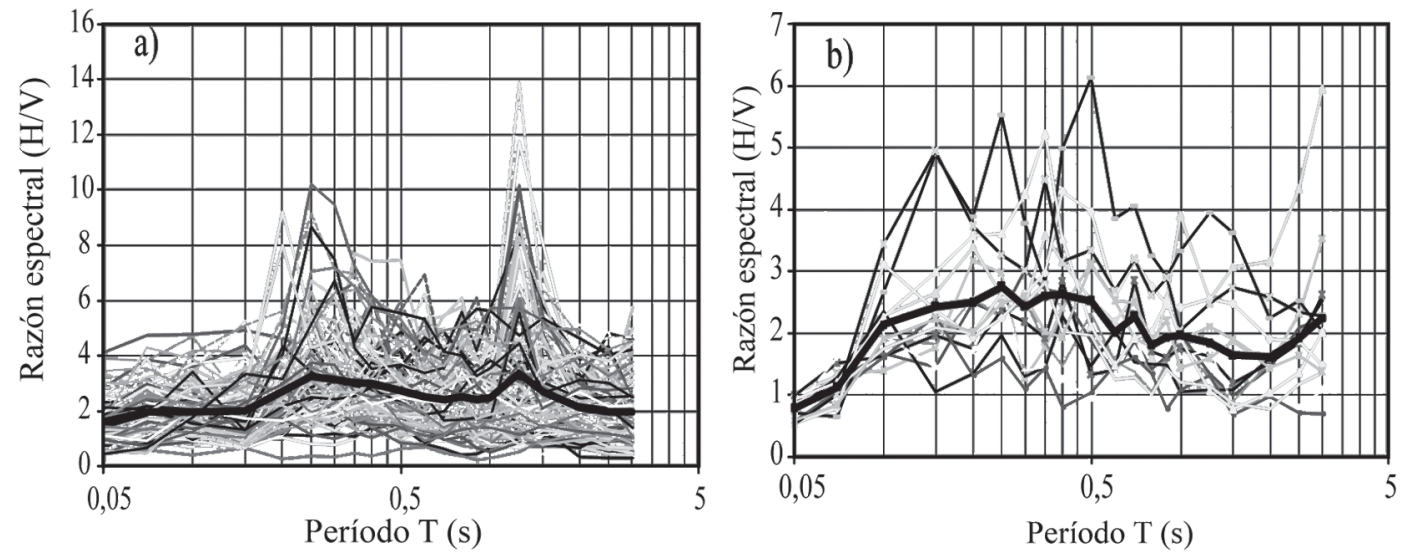

Fig. 4: Gráficos con razones espectrales que muestran dificultad para identificar To: a) muy plana y con dos picos con amplitud semejante $\mathrm{y} b$ ) no se aprecia una tendencia definida debido a la falta de registros.

B y un $15 \%$ y $32 \%$ a categorías $\mathrm{C}$ y $\mathrm{D}$, respectivamente, lo que significa que solamente un $53 \%$ de las estaciones fueron aceptablemente clasificadas, $\mathrm{o}$ sea, aproximadamente la mitad y este representa el menor porcentaje de estaciones bien calificadas de los tres países.

Para El Salvador, un 23\% de los sitios corresponde a suelo S I y un $25 \%$ a S IV, pero dentro de ambos casos, la mayoría de los sitios fueron calificados dentro de la peor categoría, la D. Por lo tanto, existen pocos sitios confiables correspondientes a suelo blando o a roca según los datos provenientes de este país.

Los niveles de amplificación de las razones espectrales promedio para El Salvador tienden a ser más bajos que los de Costa Rica y Nicaragua ya que corresponden a valores entre 2 y 3 , por lo que estos gráficos tienden a ser muy planos, lo que implica que no hay diferencias significativas entre los índices estimados para cada sitio y no fue fácil identificar $\mathrm{T}_{0}$.

Sobresalen casos como las estaciones: ESNO, ESLI, UDBS y CEUC que tienen gran cantidad de registros pero todos estos casos fueron calificados como categoría $\mathrm{C}$, por lo que no son muy confiables.

Por otro lado, la mayor parte de las estaciones calificadas según la categoría más baja (D) aportan pocos registros, entre 1 y 8 cada una, por lo que será necesario volverlas a calificar siguiendo este procedimiento en el futuro, cuando se cuente con un mayor número de registros.
Los resultados de la clasificación obtenidos se resumen en las figs. 5 y 6 .

En la fig. 5 se comparan porcentualmente las cuatro calificaciones propuestas obtenidas por los sitios en estudio. Se observa que la categoría B (estaciones bien calificadas) tiene un porcentaje casi constante en los tres países y por lo tanto, es más o menos el mismo al considerar todos los datos (última columna del gráfico).

Es evidente además el bajo porcentaje de sitios con calificación A (muy bien calificadas) para El Salvador y una considerable cantidad de emplazamientos correspondientes a ese país con la calificación más baja, la $\mathrm{D}$.

En la columna correspondiente a los totales, se observa que más de la mitad de los sitios obtuvieron una buena calificación $(\mathrm{A}+\mathrm{B})$, siendo el mayor porcentaje el correspondiente a B. Sin embargo, la presencia de lugares con calificación D es significativa, por lo que la clasificación de muchos sitios no resulta confiable.

La fig. 6 muestra la comparación porcentual de los distintos tipos de suelo en los que se clasificaron los sitios bajo estudio que obtuvieron una calificación A o B (los mejores calificados dentro de todos los estudiados). Estos corresponden a 18 emplazamientos en Nicaragua, 30 en Costa Rica y 34 en El Salvador, lo que da un total de 82 sitios, que representan un poco más de la mitad de las 134 estaciones acelerográficas consideradas inicialmente. 
Cuadro 3

Selección del tipo de suelo para las estaciones de Nicaragua

\begin{tabular}{|c|c|c|c|c|c|c|c|c|c|c|c|c|c|}
\hline \multirow[b]{2}{*}{ Estación } & \multirow[b]{2}{*}{$\begin{array}{l}\text { Geol, } \\
\text { local }\end{array}$} & \multirow[b]{2}{*}{$\begin{array}{l}\text { Mapa } \\
\text { USGS }\end{array}$} & \multirow[b]{2}{*}{$\begin{array}{c}\text { No, } \\
\text { compon, }\end{array}$} & \multicolumn{3}{|c|}{ Usando pediodo } & \multicolumn{5}{|c|}{ Usando índice } & \multirow{2}{*}{$\begin{array}{l}\text { Clas, } \\
\text { final }\end{array}$} & \multirow[b]{2}{*}{ Calific, } \\
\hline & & & & $\begin{array}{l}\text { To } \\
\text { (s) }\end{array}$ & Amplit, & $\begin{array}{c}\text { Clase } \\
\text { periodo }\end{array}$ & $\begin{array}{c}\text { SI } \\
\text { I }\end{array}$ & $\begin{array}{l}\text { SI } \\
\text { II }\end{array}$ & $\begin{array}{c}\text { SI } \\
\text { III }\end{array}$ & $\begin{array}{c}\text { SI } \\
\text { IV }\end{array}$ & $\begin{array}{l}\text { Clase } \\
\text { índice }\end{array}$ & & \\
\hline NBOA & roca & S II & 10 & 0,1 & 2,66 & S I & 0,92 & 0,79 & 0,67 & 0,66 & S I & S I & A \\
\hline $\mathrm{NCHB}$ & blando & S III & 12 & 0,7 & 4 & S IV & 0,73 & 0,77 & 0,78 & 0,8 & S III & S III & A \\
\hline $\mathrm{NCHI}$ & blando & S III & 102 & 0,4 & 4,32 & S III & 0,73 & 0,84 & 0,94 & 0,8 & S III & S III & A \\
\hline NJIN & roca & S II & 22 & 0,1 & 5,88 & S I & 0,85 & 0,77 & 0,64 & 0,59 & S I & S I & A \\
\hline NJUI & roca & S III & 12 & 0,1 & 1,5 & S I & 0,68 & 0,58 & 0,47 & 0,47 & S I & S I & A \\
\hline NLEO & blando & S III & 62 & 0,4 & 3,9 & S III & 0,72 & 0,82 & 0,83 & 0,78 & S III & S III & A \\
\hline NMAG & $? ?$ & S II & 6 & 0,35 & 3,2 & S II & 0,85 & 0,87 & 0,76 & 0,74 & S II & S II & A \\
\hline NMBA & blando & S II & 2 & 0,5 & 3,78 & S III & 0,74 & 0,8 & 0,71 & 0,73 & S II & S III & A \\
\hline NMIS & blando & S III & 4 & 0,15 & 2,85 & S IV & 0,79 & 0,74 & 0,62 & 0,61 & S I & S III & A \\
\hline NMTN & blando & S III & 6 & 0,5 & 4,29 & S III & 0,62 & 0,71 & 0,73 & 0,74 & S IV & S III & A \\
\hline NEST & blando & S III & 20 & 0,5 & 3,25 & S III & 0,75 & 0,79 & 0,69 & 0,68 & S II & S III & B \\
\hline NMAI & $? ?$ & S III & 8 & plano & plano & & 0,74 & 0,79 & 0,71 & 0,79 & S II & S III & B \\
\hline NMAT & roca & S II & 12 & plano & plano & & 0,85 & 0,76 & 0,65 & 0,64 & S I & S I & B \\
\hline NMBP & $? ?$ & S II & 2 & 1,5 & 4,14 & S IV & 0,55 & 0,62 & 0,58 & 0,63 & S II & S II & B \\
\hline NMCE & $? ?$ & S II & 6 & 0,5 & 2,76 & S III & 0,87 & 0,85 & 0,73 & 0,74 & S I & S II & B \\
\hline NREF & blando & S II & 66 & 0,15 & 3,97 & S I & 0,77 & 0,87 & 0,74 & 0,68 & S II & S II & B \\
\hline NRIV & blando & S II & 12 & 0,5 & 3,1 & S III & 0,78 & 0,85 & 0,82 & 0,8 & S II & S II & B \\
\hline NDEC & blando & S II & 68 & 0,25 & 2,4 & S II & 0,92 & 0,86 & 0,72 & 0,7 & S I & S II & B \\
\hline NCAM & $? ?$ & --- & 4 & 0,5 & 5,07 & S III & 0,62 & 0,71 & 0,84 & 0,85 & S IV & S III & $\mathrm{C}$ \\
\hline NGRA & blando & S III & 14 & 0,25 & 2,74 & S II & 0,86 & 0,88 & 0,73 & 0,68 & S II & S II & $\mathrm{C}$ \\
\hline NJMP & $? ?$ & S II & 4 & 0,1 & 2,7 & S I & 0,79 & 0,68 & 0,59 & 0,54 & S I & S I & $\mathrm{C}$ \\
\hline NBNA & $? ?$ & --- & 2 & 0,8 & 4,02 & S IV & 0,75 & 0,79 & 0,76 & 0,72 & S II & S III & $\mathrm{D}$ \\
\hline NINE & blando & S III & 50 & 0,2 & 2,67 & S II & 0,94 & 0,84 & 0,68 & 0,66 & S I & S II & $\mathrm{D}$ \\
\hline NMAS & blando & S II & 8 & 0,7 & 2,74 & S IV & 0,84 & 0,81 & 0,7 & 0,69 & S I & S II & D \\
\hline NMCC & blando & S II & 2 & Plano & plano & S IV & 0,71 & 0,82 & 0,78 & 0,81 & S II & S II & $\mathrm{D}$ \\
\hline NJUA & $? ?$ & -- & 4 & Plano & plano & & 0,86 & 0,82 & 0,67 & 0,67 & S I & S I & $\mathrm{D}$ \\
\hline
\end{tabular}

Se observa la poca presencia de sitios S IV (suelo blando) y de S I (roca), esto último principalmente para el caso de Costa Rica. Es notable además el predominio de lugares clasificados como S II (suelo firme) en El Salvador y de S III (suelo medio) en Nicaragua.

Respecto a los totales, es evidente el predominio de suelos S II y S III y la poca presencia de sitios con suelos clasificados como S I y S IV.

Al comparar el mapa de la fig. 1 con los resultados obtenidos siguiendo la metodología de clasificación propuesta, se puede afirmar que las rocas volcánicas del Cenozoico, que es donde se ubican la mayoría de las estaciones acelerográficas según el mapa mencionado, se clasifican desde el punto de vista geotécnico como suelos S II (firme) y S III (medio) según el NEHRP (BSSC, 2003).

Posiblemente, estas dos categorías de suelo que predominan en este tipo de roca están relacionadas con el espesor de ese material hasta llegar a la roca competente, de manera que S III 
Cuadro 4

Selección del tipo de suelo para las estaciones de Costa Rica

\begin{tabular}{|c|c|c|c|c|c|c|c|c|c|c|c|c|c|}
\hline \multirow[b]{2}{*}{ Estac, } & \multirow[b]{2}{*}{$\begin{array}{l}\text { Geol, } \\
\text { local }\end{array}$} & \multirow[b]{2}{*}{$\begin{array}{l}\text { Mapa } \\
\text { USGS }\end{array}$} & \multirow[b]{2}{*}{$\begin{array}{c}\text { No, } \\
\text { compon, }\end{array}$} & \multicolumn{3}{|c|}{ Usando periodo } & \multicolumn{5}{|c|}{ Usando índice } & \multirow[b]{2}{*}{$\begin{array}{l}\text { Clas, } \\
\text { final }\end{array}$} & \multirow[b]{2}{*}{ Calific, } \\
\hline & & & & To (s) & Amplit, & $\begin{array}{c}\text { Clase } \\
\text { periodo }\end{array}$ & $\begin{array}{l}\text { SI } \\
\text { I }\end{array}$ & $\begin{array}{l}\text { SI } \\
\text { II }\end{array}$ & $\begin{array}{l}\text { SI } \\
\text { III }\end{array}$ & $\begin{array}{l}\text { SI } \\
\text { IV }\end{array}$ & $\begin{array}{l}\text { Clase } \\
\text { índice }\end{array}$ & & \\
\hline AALJ & blando & S II & 82 & 0,9 & 4,01 & SC IV & 0,7 & 0,76 & 0,77 & 0,87 & S IV & S IV & A \\
\hline CCTG & blando & S II & 44 & 0,8 & 3,27 & SC IV & 0,73 & 0,85 & 0,87 & 0,8 & S III & S III & A \\
\hline SDES & firme & S III & 48 & 0,25 & 4,6 & SC II & 0,72 & 0,8 & 0,71 & 0,62 & S II & S II & A \\
\hline AFIR & firme & S II & 34 & 0,25 & 3,07 & SC II & 0,81 & 0,9 & 0,75 & 0,7 & S II & S II & A \\
\hline SFRA & firme & S II & 150 & 0,25 & 3,23 & SC II & 0,77 & 0,91 & 0,84 & 0,8 & S II & S II & A \\
\hline PGLF & roca & S II & 68 & 0,4 & 2,6 & SC II & 0,81 & 0,92 & 0,78 & 0,77 & S II & S II & A \\
\hline SGTS & blando & S II & 30 & 0,65 & 4,07 & SC IV & 0,76 & 0,84 & 0,89 & 0,83 & S III & S III & A \\
\hline SGUA & firme & S II & 58 & 0,2 & 4,46 & SC II & 0,75 & 0,85 & 0,75 & 0,71 & S II & S II & A \\
\hline SHAT & blando & S III & 50 & 0,4 & 3,17 & SC III & 0,76 & 0,87 & 0,85 & 0,83 & S III & S III & A \\
\hline HMGL & blando & S II & 18 & 0,6 & 3,92 & SC IV & 0,73 & 0,75 & 0,73 & 0,71 & S III & S III & A \\
\hline SNFL & roca & S II & 142 & 0,2 & 4,94 & SC I & 0,75 & 0,83 & 0,72 & 0,63 & S II & S II & A \\
\hline CPAR & blando & S II & 6 & 0,3 & 5,05 & SC II & 0,74 & 0,81 & 0,81 & 0,69 & S III & S III & A \\
\hline RMOI & blando & S III & 34 & 0,6 & 2,62 & SC IV & 0,76 & 0,85 & 0,77 & 0,8 & S II & S III & A \\
\hline ASRM & blando & S II & 60 & 1,25 & 3,66 & SC IV & 0,73 & 0,75 & 0,67 & 0,75 & S IV & S IV & A \\
\hline GSTC & blando & S III & 16 & 0,5 & 4,78 & SC III & 0,72 & 0,83 & 0,90 & 0,73 & S III & S III & A \\
\hline CTEC & blando & S II & 12 & 0,6 & 3,22 & SC IV & 0,78 & 0,83 & 0,84 & 0,83 & S IV & S IV & A \\
\hline PBJU & $? ?$ & S III & 4 & 0,3 & 5,42 & SC II & 0,65 & 0,76 & 0,82 & 0,78 & S III & S III & B \\
\hline SCAR & blando & S III & 72 & 0,8 & 3,04 & SC IV & 0,8 & 0,87 & 0,83 & 0,82 & S II & S III & B \\
\hline $\mathrm{CCCH}$ & roca & S I & 28 & 0,4 & 2,0 & SC II & 0,81 & 0,75 & 0,63 & 0,64 & S I & S I & B \\
\hline $\mathrm{CCDN}$ & firme & S II & 188 & 0,5 & 3,54 & SC III & 0,72 & 0,77 & 0,74 & 0,7 & S II & S II & B \\
\hline SECA & blando & S III & 136 & 0,5 & 3,53 & SC III & 0,76 & 0,86 & 0,81 & 0,72 & S II & S III & B \\
\hline SHTO & blando & S III & 44 & 0,4 & 2,56 & SC III & 0,83 & 0,88 & 0,78 & 0,8 & S II & S III & B \\
\hline LLIM & firme & S II & 10 & 0,2 & 3,76 & SC II & 0,85 & 0,83 & 0,69 & 0,63 & S I & S II & B \\
\hline SLPF & blando & S III & 132 & 0,35 & 4,18 & SC II & 0,72 & 0,86 & 0,92 & 0,8 & S III & S III & B \\
\hline HOVS & firme & S II & 56 & 0,2 & 3,08 & SC I & 0,82 & 0,82 & 0,70 & 0,68 & S II & S II & B \\
\hline RGAR & blando & S II & 20 & 0,3 & 2,93 & SC II & 0,84 & 0,86 & 0,75 & 0,7 & S II & S II & B \\
\hline CTBA & firme & S II & 19 & 0,1 & 3,17 & SC I & 0,9 & 0,78 & 0,66 & 0,64 & S I & S II & B \\
\hline SISD & blando & S II & 132 & 0,3 & 3,83 & SC II & 0,88 & 0,87 & 0,72 & 0,63 & S II & S II & B \\
\hline PQSP & $? ?$ & S III & 90 & 0,7 & 3,12 & SC IV & 0,71 & 0,8 & 0,77 & 0,78 & S II & S III & B \\
\hline CSLG & blando & S II & 54 & 0,35 & 4,85 & SC II & 0,66 & 0,76 & 0,83 & 0,75 & S III & S III & B \\
\hline SPCL & blando & S II & 34 & 0,35 & 2,18 & SC II & 0,78 & 0,66 & 0,52 & 0,54 & S I & S II & $\mathrm{C}$ \\
\hline PQPS & $? ?$ & S II & 48 & 0,1 & 3,27 & SC I & 0,9 & 0,88 & 0,76 & 0,66 & S I & S I & $\mathrm{C}$ \\
\hline RALT & firme & S II & 22 & 0,7 & 3,69 & SC IV & 0,7 & 0,78 & 0,80 & 0,85 & S IV & S IV & $\mathrm{C}$ \\
\hline RBAR & firme & S III & 2 & 0,5 & 4,45 & SC III & 0,66 & 0,69 & 0,66 & 0,64 & S II & S III & $\mathrm{C}$ \\
\hline CRCP & firme & S II & 12 & 0,65 & 4,32 & SC IV & 0,69 & 0,74 & 0,82 & 0,84 & S IV & S IV & $\mathrm{C}$ \\
\hline SROH & blando & S II & 24 & 0,8 & 2,85 & SC IV & 0,78 & 0,87 & 0,81 & 0,79 & S II & S II & $\mathrm{C}$ \\
\hline
\end{tabular}


Cuadro 4 (continuación)

Selección del tipo de suelo para las estaciones de Costa Rica

\begin{tabular}{|c|c|c|c|c|c|c|c|c|c|c|c|c|c|}
\hline \multirow[b]{2}{*}{ Estac, } & \multirow[b]{2}{*}{$\begin{array}{l}\text { Geol, } \\
\text { local }\end{array}$} & \multirow[b]{2}{*}{$\begin{array}{l}\text { Mapa } \\
\text { USGS }\end{array}$} & \multirow[b]{2}{*}{$\begin{array}{c}\text { No, } \\
\text { compon, }\end{array}$} & \multicolumn{3}{|c|}{ Usando periodo } & \multicolumn{5}{|c|}{ Usando índice } & \multirow[b]{2}{*}{$\begin{array}{l}\text { Clas, } \\
\text { final }\end{array}$} & \multirow[b]{2}{*}{ Calific, } \\
\hline & & & & To (s) & Amplit, & $\begin{array}{c}\text { Clase } \\
\text { periodo }\end{array}$ & $\begin{array}{c}\text { SI } \\
\text { I }\end{array}$ & $\begin{array}{l}\text { SI } \\
\text { II }\end{array}$ & $\begin{array}{l}\text { SI } \\
\text { III }\end{array}$ & $\begin{array}{l}\text { SI } \\
\text { IV }\end{array}$ & $\begin{array}{l}\text { Clase } \\
\text { índice }\end{array}$ & & \\
\hline RTUR & firme & S II & 8 & 0,15 & 3,38 & SC I & 0,87 & 0,79 & 0,66 & 0,64 & S I & S I & $\mathrm{C}$ \\
\hline SGEO & blando & S III & 4 & 0,35 & 3,25 & SC II & 0,72 & 0,82 & 0,73 & 0,71 & S II & S II & $\mathrm{D}$ \\
\hline GLIB & firme & --- & 4 & 1 & 3,47 & SC IV & 0,72 & 0,73 & 0,67 & 0,7 & S II & S II & $\mathrm{D}$ \\
\hline GNYA & blando & S II & 6 & 0,5 & 1,57 & SC III & 0,59 & 0,49 & 0,38 & 0,4 & S I & S II & $\mathrm{D}$ \\
\hline SSAB & blando & S II & 2 & 0,35 & 3,61 & SC II & 0,72 & 0,67 & 0,56 & 0,5 & S I & S II & $\mathrm{D}$ \\
\hline ASCS & Blando & S II & & ---- & & & 0,81 & 0,72 & 0,63 & 0,6 & S I & & $\mathrm{D}$ \\
\hline PTGA & blando & S III & 4 & 0,9 & 1,88 & SC IV & 0,73 & 0,65 & 0,51 & 0,51 & S I & S III & $\mathrm{D}$ \\
\hline
\end{tabular}

corresponda a suelos de mayor potencia que los sitios donde se identificó S II. Sin embargo, hasta que no se cuente con pruebas geofísicas suficientes, no se podrá ratificar esta conclusión.

\section{COMPARACIÓN DE LAS RAZONES ESPECTRALES PROMEDIO OBTENIDAS PARA AMÉRICACENTRAL CON LAS DE JAPÓN}

Debido a que la metodología de clasificación propuesta en el presente estudio es muy semejante a la seguida para Japón según Zhao et al. (2006), se consideró conveniente comparar los resultados obtenidos en ambos estudios a modo referencia.

Sin embargo, es necesario tomar en cuenta que se están comparando regiones del mundo con geologías muy distintas, sobretodo porque en Japón si es común hallar rocas aflorantes competentes (GSJ, 2010), por lo que una buena cantidad de emplazamientos utilizados por Zhao et al. (2006) se ubican en esta condición.

La fig. 7 compara las razones espectrales promedio obtenidas para Japón (fig. 7a) y para América Central (fig. 7b), para los cuatro tipos de suelo considerados. Para esta comparación, solamente fueron consideradas las estaciones con calificación A y B de El Salvador, Nicaragua y Costa Rica.

En términos generales, se observa una semejanza en cuanto a las tendencias mostradas por las cuatro curvas obtenidas en ambos estudios. Sin embargo, las razones tienen amplificaciones mayores para Japón. Además, las curvas obtenidas de ese país parecen ser más puntiagudas (definen mejor los periodos de máxima amplificación).

La fig. 8a compara las razones espectrales para suelo S I (roca). Se observa que la curva correspondiente a América Central es muy plana, con una amplificación casi constante igual a 1,5 en todo el rango de periodos. En cambio, la que corresponde a Japón muestra claramente un pico en un periodo de $0,15 \mathrm{~s}$ con una amplitud asociada de 2,5 y luego decrece. En ese valor de periodo es en el que se observa la mayor diferencia entre las dos razones espectrales, resultado que la de Japón es aproximadamente 1,85 veces mayor que la de América Central para ese periodo específico.

La fig. 8b presenta las razones espectrales para suelo S II (firme). Nuevamente, la que corresponde a Japón muestra amplificaciones mayores en todo el rango de periodos, sobretodo alrededor de $0,2 \mathrm{~s}$, donde se identifica un factor de 1,36 de diferencia entre ambas curvas. A partir de $0,5 \mathrm{~s}$ hasta $3 \mathrm{~s}$ (periodos largos), las curvas son semejantes.

La comparación entre las razones espectrales para suelo S III (medio) es mostrada en la fig. 9a. Existe una gran semejanza entre ambas curvas de $0,05 \mathrm{~s}$ a $0,2 \mathrm{~s}$ (periodos cortos) y alguna similitud de $0,7 \mathrm{~s}$ a $3 \mathrm{~s}$ (periodos largos), pero entre $0,2 \mathrm{y}$ 0,7 s la curva que se obtuvo para Japón es bastante superior a la obtenida para América Central, llegándose a un factor de diferencia entre ambas de 1,37 en $0,4 \mathrm{~s}$ de periodo. 
Cuadro 5

Selección del tipo de suelo para las estaciones de El Salvador

\begin{tabular}{|c|c|c|c|c|c|c|c|c|c|c|c|c|c|}
\hline \multirow[b]{2}{*}{ Estac. } & \multirow[b]{2}{*}{$\begin{array}{l}\text { Geol. } \\
\text { local }\end{array}$} & \multirow[b]{2}{*}{$\begin{array}{l}\text { Mapa } \\
\text { USGS }\end{array}$} & \multirow[b]{2}{*}{$\begin{array}{c}\text { No. } \\
\text { compon. }\end{array}$} & \multicolumn{3}{|c|}{ Usando periodo } & \multicolumn{5}{|c|}{ Usando índice } & \multirow[b]{2}{*}{$\begin{array}{l}\text { Clas. } \\
\text { final }\end{array}$} & \multirow[b]{2}{*}{ Calific } \\
\hline & & & & $\begin{array}{l}\text { To } \\
\text { (s) }\end{array}$ & Amplit. & $\begin{array}{c}\text { Clase } \\
\text { periodo }\end{array}$ & SII & SIII & SI III & SIIV & $\begin{array}{l}\text { Clase } \\
\text { índice }\end{array}$ & & \\
\hline ESBN & firme & --- & 6 & 0,25 & 2,7 & S II & 0,77 & 0,72 & 0,63 & 0,65 & S I & S II & A \\
\hline ESTE & firme & S II & 66 & 0,2 & 3,53 & S II & 0,87 & 0,86 & 0,69 & 0,66 & S II & S II & A \\
\hline HERR & blando & S III & 64 & 1,5 & 6,39 & S IV & 0,4301 & 0,5371 & 0,6448 & 0,6426 & S IV & S IV & A \\
\hline PERQ & firme & $\begin{array}{l}\text { S I - } \\
\text { S II }\end{array}$ & 8 & 0,4 & 4,06 & S II & 0,769 & 0,8369 & 0,825 & 0,7425 & S II & S II & A \\
\hline UCHI & firme & S II & 74 & 0,2 & 2,46 & S II & 0,8265 & 0,8172 & 0,6749 & 0,6639 & S II & S II & A \\
\hline UESS & firme & S II & 174 & 0,35 & 4,66 & S II & 0,6843 & 0,7822 & 0,7569 & 0,6731 & S II & S II & A \\
\hline AEIS & blando & S III & 190 & 0,8 & 3,8 & S IV & 0,48 & 0,4 & 0,32 & 0,33 & S IV & S IV & A \\
\hline EMAS & roca & $\begin{array}{l}\text { S I - } \\
\text { S II }\end{array}$ & 6 & 0,15 & 2,38 & S I & 0,8419 & 0,7504 & 0,638 & 0,6044 & S I & S I & A \\
\hline ESEX & blando & S II & 64 & 0,6 & 3 & S IV & 0,69 & 0,77 & 0,7 & 0,7 & S II & S III & A \\
\hline LUNA & blando & $\begin{array}{l}\text { S II - } \\
\text { S III }\end{array}$ & 52 & 1,5 & 3,08 & S IV & 0,7468 & 0,7717 & 0,7044 & 0,8014 & S II & S II & B \\
\hline MAGT & firme & $\begin{array}{l}\text { S II - } \\
\text { S III }\end{array}$ & 116 & 1,25 & 2,73 & S IV & 0,7516 & 0,8148 & 0,7581 & 0,7805 & S II & S II & B \\
\hline ESDB & firme & --- & 10 & 0,9 & 3,15 & S IV & 0,72 & 0,72 & 0,67 & 0,67 & S II & S II & B \\
\hline ESOB & firme & --- & 50 & 0,6 & 2,21 & S IV & 0,78 & 0,74 & 0,6 & 0,6 & S II & S II & B \\
\hline ESPA & firme & S II & 24 & 0,5 & 2,48 & S III & 0,84 & 0,87 & 0,74 & 0,73 & S II & S II & B \\
\hline ESRF & roca & --- & 6 & 0,9 & 2,53 & S IV & 0,76 & 0,72 & 0,62 & 0,61 & S I & S I & B \\
\hline ESSA & firme & S II & 6 & $\begin{array}{c}\text { pla- } \\
\text { no }\end{array}$ & plano & plano & 0,78 & 0,71 & 0,59 & 0,59 & S I & S II & B \\
\hline ESST & firme & --- & 4 & 0,4 & 3,9 & S III & 0,76 & 0,86 & 0,84 & 0,71 & S II & S II & B \\
\hline ESTO & blando & S II & 58 & 0,7 & 2,64 & S IV & 0,75 & 0,8 & 0,74 & 0,73 & S II & S II & B \\
\hline ESUC & firme & --- & 6 & 0,7 & 3,17 & S IV & 0,75 & 0,74 & 0,66 & 0,67 & S II & S II & B \\
\hline ESZA & firme & S II & 64 & 1 & 2,68 & S IV & 0,69 & 0,76 & 0,7 & 0,71 & S II & S II & B \\
\hline SEMS & firme & --- & 6 & 0,25 & 2,84 & S II & 0,84 & 0,75 & 0,62 & 0,59 & S I & S I & B \\
\hline SMIG & firme & S III & 88 & 0,25 & 2,54 & S II & 0,793 & 0,7277 & 0,6056 & 0,6169 & S I & S I & B \\
\hline SNET & blando & S II & 196 & 0,5 & 2,86 & S III & 0,7799 & 0,8031 & 0,6774 & 0,6879 & S II & S II & B \\
\hline SONS & blando & S II & 202 & 0,1 & 2,3 & S I & 0,8003 & 0,82 & 0,7022 & 0,6806 & S II & S II & B \\
\hline UNCO & firme & S II & 68 & 0,7 & 2,38 & S IV & 0,7431 & 0,7945 & 0,654 & 0,6566 & S II & S II & B \\
\hline CEME & roca & --- & 2 & plano & plano & plano & 0,69 & 0,65 & 0,55 & 0,61 & S I & S I & B \\
\hline CHIN & firme & S II & 80 & 1,5 & 2,12 & S IV & 0,7748 & 0,7393 & 0,6198 & 0,6102 & S II & S II & B \\
\hline CPRF & firme & --- & 16 & 0,3 & 2,3 & S II & 0,8 & 0,69 & 0,57 & 0,56 & S I & S II & B \\
\hline CPRS & roca & --- & 14 & 0,2 & 1,73 & S II & 0,77 & 0,66 & 0,54 & 0,55 & S I & S I & B \\
\hline ECHB & roca & $\begin{array}{l}\text { S I - } \\
\text { S II }\end{array}$ & 8 & 0,5 & 2,9 & S III & 0,7753 & 0,8533 & 0,8011 & 0,8143 & S II & S II & B \\
\hline ECIG & firme & $\begin{array}{l}\text { S II - } \\
\text { S III }\end{array}$ & 12 & 0,8 & 2,4 & S IV & 0,7799 & 0,7827 & 0,6683 & 0,6983 & S II & S II & B \\
\hline
\end{tabular}


Cuadro 5 (continuación)

Selección del tipo de suelo para las estaciones de El Salvador

\begin{tabular}{|c|c|c|c|c|c|c|c|c|c|c|c|c|c|}
\hline \multirow[b]{2}{*}{ Estac. } & \multirow[b]{2}{*}{$\begin{array}{c}\text { Geol. } \\
\text { local }\end{array}$} & \multirow[b]{2}{*}{$\begin{array}{l}\text { Mapa } \\
\text { USGS }\end{array}$} & \multirow[b]{2}{*}{$\begin{array}{c}\text { No. } \\
\text { compon. }\end{array}$} & \multicolumn{3}{|c|}{ Usando periodo } & \multicolumn{5}{|c|}{ Usando índice } & \multirow[b]{2}{*}{$\begin{array}{l}\text { Clas. } \\
\text { final }\end{array}$} & \multirow[b]{2}{*}{ Calific. } \\
\hline & & & & $\begin{array}{l}\text { To } \\
\text { (s) }\end{array}$ & Amplit. & $\begin{array}{c}\text { Clase } \\
\text { periodo }\end{array}$ & SII & SI II & SI III & SIIV & $\begin{array}{l}\text { Clase } \\
\text { índice }\end{array}$ & & \\
\hline EJUA & $? ?$ & S II & 68 & 0.9 & 2,89 & S IV & 0,6902 & 0,7414 & 0,6849 & 0,6849 & S II & S II & B \\
\hline ESBA & blando & S II & 60 & 1.5 & 2,25 & S IV & 0,8 & 0,78 & 0,65 & 0,67 & S I & S III & B \\
\hline ESGR & $? ?$ & S II & 112 & 2 & 2,31 & S IV & 0,8 & 0,8 & 0,68 & 0,7 & S II & S II & B \\
\hline ESCI & $? ?$ & --- & 8 & 1.25 & 2,94 & S IV & 0,76 & 0,81 & 0,73 & 0,82 & S IV & S IV & $\mathrm{C}$ \\
\hline ESLI & blando & $\begin{array}{l}\text { S II - } \\
\text { S III }\end{array}$ & 68 & 0.2 & 4 & S II & 0,79 & 0,9 & 0,77 & 0,71 & S II & S II & $\mathrm{C}$ \\
\hline ESNO & blando & $\begin{array}{l}\text { S I - } \\
\text { S II }\end{array}$ & 148 & 0.4 & 2,8 & $\begin{array}{c}\text { S II - S } \\
\text { III }\end{array}$ & 0,72 & 0,82 & 0,76 & 0,76 & S II & S II & $\mathrm{C}$ \\
\hline ESSV & $? ?$ & --- & 14 & 0.8 & $\begin{array}{c}1,8 \\
\text { plano }\end{array}$ & plano & 0,8 & 0,72 & 0,6 & 0,63 & S I & S I & $\mathrm{C}$ \\
\hline ESVF & $? ?$ & --- & 8 & 0.3 & 2,02 & S II & 0,66 & 0,64 & 0,54 & 0,6 & S II & S II & $\mathrm{C}$ \\
\hline ESVS & $? ?$ & --- & 8 & 1 & 3,73 & S IV & 0,61 & 0,67 & 0,64 & 0,74 & S IV & S IV & C \\
\hline SJAC & blando & --- & 12 & 0.3 & 3,82 & S II & 0,81 & 0,78 & 0,65 & 0,6 & S I & S II & C \\
\hline UDBS & blando & $\begin{array}{l}\text { S II - } \\
\text { S III }\end{array}$ & 130 & 0.4 & 3,44 & S II & 0,7722 & 0,767 & 0,6659 & 0,6673 & S II & S II & C \\
\hline ACAJ & firme & S III & 28 & 1.25 & 3,53 & S IV & 0,6085 & 0,6782 & 0,6631 & 0,8118 & S IV & S IV & C \\
\hline CEUC & firme & $\begin{array}{l}\text { S II - } \\
\text { S III }\end{array}$ & 62 & 0.7 & 2,95 & S IV & 0,7177 & 0,7903 & 0,7646 & 0,8455 & S IV & S IV & $\mathrm{C}$ \\
\hline ESAH & roca & --- & 4 & 0.5 & 1,82 & S III & 0,76 & 0,69 & 0,56 & 0,56 & S I & S I & D \\
\hline ESAI & $? ?$ & --- & 6 & 0.6 & 2,63 & S IV & 0,77 & 0,76 & 0,64 & 0,64 & S II & S IV & D \\
\hline ESAS & blando & --- & 2 & 1.5 & 4,89 & S IV & 0,61 & 0,67 & 0,68 & 0,78 & S IV & S IV & D \\
\hline ESAT & $? ?$ & --- & 4 & 0.7 & 3 & S IV & 0,77 & 0,76 & 0,66 & 0,66 & S I & S IV & D \\
\hline ESCF & roca & --- & 2 & 2 & 4,1 & S II & 0,8 & 0,77 & 0,67 & 0,71 & S I & S I & D \\
\hline ESCS & $? ?$ & --- & 4 & 2 & 3,82 & S IV & 0,77 & 0,77 & 0,72 & 0,79 & S IV & S IV & $\mathrm{D}$ \\
\hline ESCU & firme & --- & 4 & 0.6 & 2,93 & S IV & 0,74 & 0,8 & 0,72 & 0,8 & S II & S IV & $\mathrm{D}$ \\
\hline ESIG & $? ?$ & --- & 2 & 0.5 & 3,24 & S III & 0,68 & 0,65 & 0,54 & 0,54 & S II & S III & D \\
\hline ESIS & $? ?$ & --- & 2 & 1.25 & 3,85 & S IV & 0,68 & 0,72 & 0,67 & 0,78 & S IV & S IV & D \\
\hline ESIV & $? ?$ & --- & 2 & 1.25 & 3,2 & S IV & 0,74 & 0,75 & 0,65 & 0,65 & S II & S IV & D \\
\hline ESMT & $? ?$ & --- & 8 & 1.5 & 2,54 & S IV & 0,82 & 0,77 & 0,66 & 0,69 & S I & S I & D \\
\hline ESRS & firme & --- & 16 & 1 & 2,54 & S IV & 0,78 & 0,81 & 0,7 & 0,7 & S II & S II & $\mathrm{D}$ \\
\hline ESSF & $? ?$ & --- & 6 & 1.25 & 2,29 & S IV & 0,49 & 0,44 & 0,37 & 0,34 & S I & S IV & D \\
\hline ESSM & $? ?$ & --- & 14 & 0.25 & 1,9 & S II & 0,81 & 0,7 & 0,54 & 0,58 & S I & S I & $\mathrm{D}$ \\
\hline ESSS & firme & --- & 8 & 1.25 & 3,6 & S IV & 0,69 & 0,68 & 0,6 & 0,63 & S I & S IV & D \\
\hline ESTC & $? ?$ & --- & 4 & 0.8 & 2,81 & S IV & 0,72 & 0,71 & 0,61 & 0,6 & S I & S IV & D \\
\hline ESUS & $? ?$ & --- & 4 & 0.6 & 2,4 & S IV & 0,8 & 0,74 & 0,61 & 0,63 & S I & S I & D \\
\hline
\end{tabular}


Cuadro 5 (continuación)

Selección del tipo de suelo para las estaciones de El Salvador

\begin{tabular}{cccc|ccc|ccccccc}
\hline & & & & \multicolumn{7}{c|}{ Usando periiodo } & \multicolumn{5}{c}{ Usando ìndice } & \\
\hline Estac. & $\begin{array}{c}\text { Geol. } \\
\text { local }\end{array}$ & $\begin{array}{c}\text { Mapa } \\
\text { USGS }\end{array}$ & $\begin{array}{c}\text { No. } \\
\text { compon. }\end{array}$ & $\begin{array}{c}\text { To } \\
\text { (s) }\end{array}$ & Amplit. & $\begin{array}{c}\text { Clase } \\
\text { periodo }\end{array}$ & SII & SI II & SI III & SI IV & $\begin{array}{c}\text { Clase } \\
\text { índice }\end{array}$ & $\begin{array}{c}\text { Clas. } \\
\text { final }\end{array}$ & Calific. \\
\hline SEMF & roca & --- & 4 & 0,25 & 2,78 & S II & 0,78 & 0,7 & 0,57 & 0,55 & S I & S I & D \\
STAN & $? ?$ & S II & 8 & 0,7 & 2,62 & S IV & 0,7048 & 0,7591 & 0,6626 & 0,6695 & S II & S II & D \\
EHGZ & roca & $\begin{array}{c}\text { S I - } \\
\text { S II }\end{array}$ & 4 & $\begin{array}{c}\text { pla- } \\
\text { no }\end{array}$ & plano & Plano & 0,46 & 0,38 & 0,3 & 0,3 & S I & S I & D \\
ESBO & blando & $\begin{array}{c}\text { S I - } \\
\text { S II }\end{array}$ & 10 & 0,25 & 3,02 & S II & 0,8978 & 0,7964 & 0,6781 & 0,6143 & S I & S I & D \\
\hline
\end{tabular}

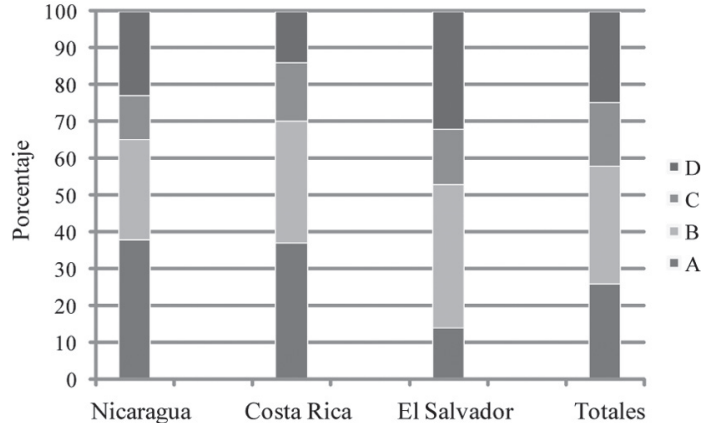

Fig. 5: Comparación porcentual entre las distintas calificaciones obtenidas por las estaciones acelerográficas de América Central. A corresponde a la calificación más alta y $\mathrm{D}$ a la más baja.

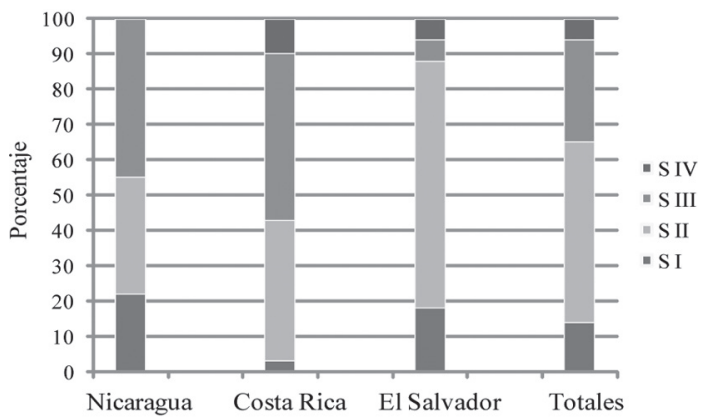

Fig. 6: Comparación porcentual de los distintos tipos de suelo identificados en las estaciones con calificación A y B (sitios mejor calificados).

Finalmente, en la fig. 9b se comparan las razones espectrales para suelo S IV (blando) y se observa que son bastante semejantes, siempre ubicándose la de Japón por encima de la de América Central. La diferencia mayor entre ambas se da en $0,7 \mathrm{~s}$ de periodo, correspondiente a un factor de 1,2 .

\section{CONCLUSIONES}

Se lograron clasificar 134 estaciones acelerográficas de América Central con base en el cálculo de razones espectrales $\mathrm{H} / \mathrm{V}$, lo que permitió estimar el periodo fundamental $\mathrm{T}_{0} \mathrm{y}$ el índice de clasificación SIK para cada sitio. Asimismo, se tomaron en cuenta otros criterios de clasificación disponibles en la bibliografía, basados en interpretaciones de mapas geológicos y en correlaciones de Vs30 con la pendiente del terreno. Todo esto permitió asignarle una calificación a cada sitio previamente clasificado.

Del total de las 134 estaciones consideradas, un $26 \%$ cumple con la coincidencia de los tres criterios de clasificación, por lo que recibieron una calificación A. Un $32 \%$ obtuvo calificación B, un $17 \%$ como $\mathrm{C}$ y un $25 \%$ como D. Esto significa que un $58 \%$ del total de las estaciones provenientes de Nicaragua, El Salvador y Costa Rica obtuvieron una clasificación aceptable (categorías A y B) y un $42 \%$ no la tienen (categorías C y D).

En general, existen pocas estaciones bien calificadas correspondientes a roca (S I) y también pocas del tipo blando (S IV), tanto bien calificadas 

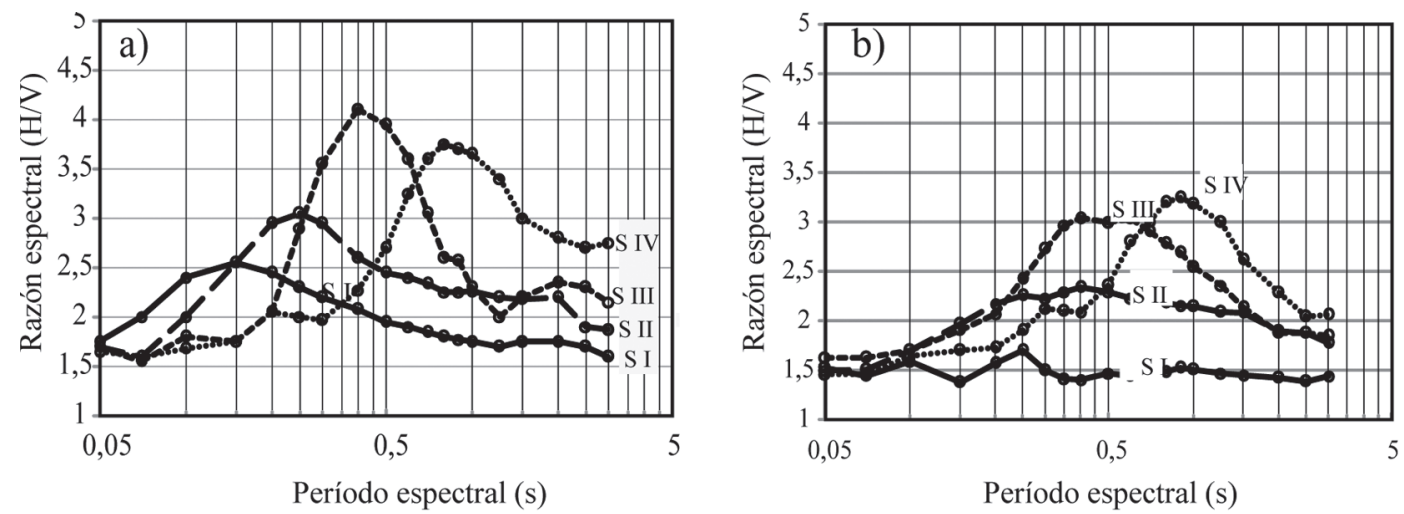

Fig. 7: Razones espectrales promedio para: a) Japón y b) América Central.
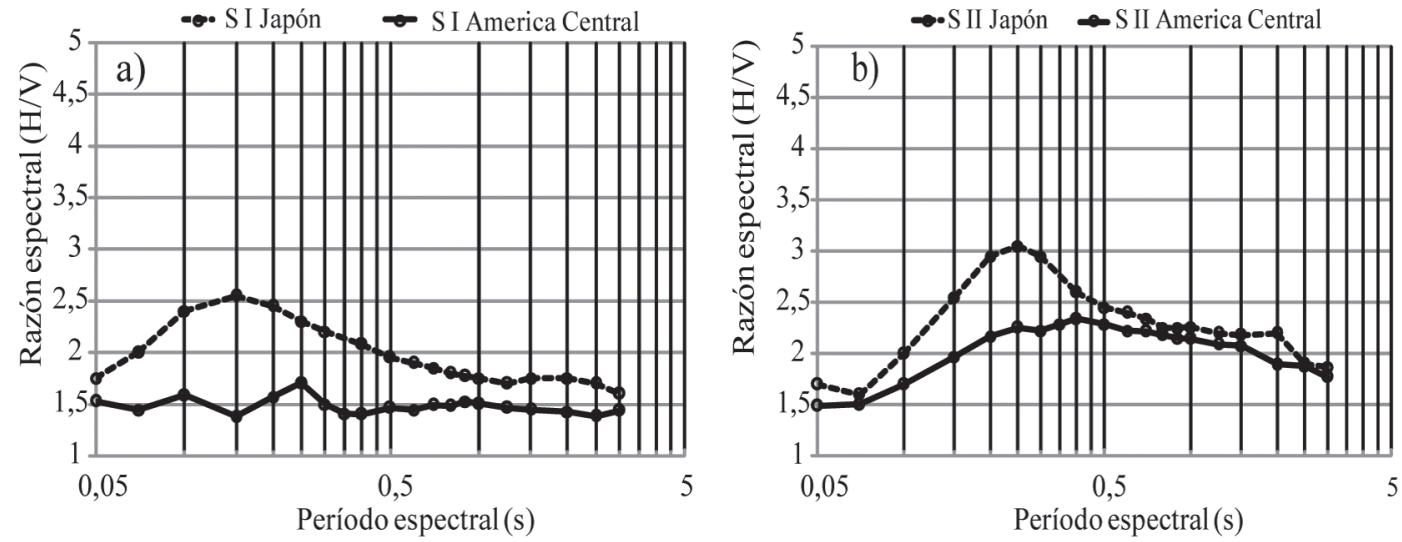

Fig. 8: Comparación de razones espectrales para distintos tipos de suelo: a) S I (roca) y b) S II (suelo firme).
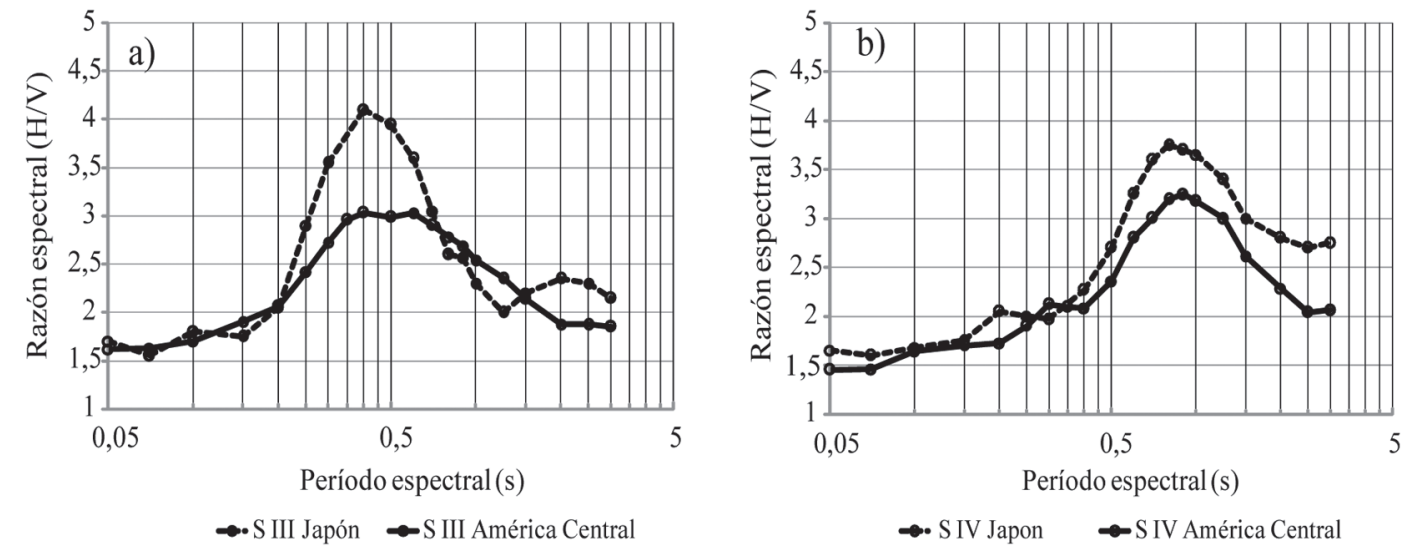

Fig. 9: Comparación de razones espectrales para distintos tipos de suelo: a) S III (medio) y b) S IV (suelo blando). 
(A y B) como si no se toma en cuenta criterio de calificación alguno.

El hecho de que pocas estaciones hayan sido clasificadas como roca es concordante con el tipo de geología predominante en los tres países, sobretodo en las zonas donde se ubica la mayor cantidad de los instrumentos (valles, zonas costeras, zonas inundables) donde predominan rocas volcánicas del Cenozoico (Bundschuh \& Alvarado, 2007). Esto hace ver la necesidad de colocar en el futuro un número mayor de acelerógrafos en condiciones bien calificadas de roca, lo que permitirá tener una mejor cobertura de registros en los cuatro tipos de suelos considerados, y no un sesgo a tipos $\mathrm{S}$ II y S III como es el caso actual, situación que dificulta obtener ecuaciones de atenuación precisas según las condiciones del sitio de registro.

Fue encontrado un porcentaje similar de estaciones satisfactoriamente clasificadas (categorías A y B) para Costa Rica y Nicaragua (aproximadamente un 64\% en ambos casos), e inferior para El Salvador (un 52\%). La clase A para El Salvador (un 14\%) es muy inferior que la misma clase para Costa Rica (un 37\%) y para Nicaragua (un 38\%).

El porcentaje de estaciones que recibieron peor calificación para Costa Rica y para Nicaragua (categorías C y D, un $36 \%$ aproximadamente) fue inferior que el obtenido en estas mismas categorías para El Salvador (un 47\%), lo que permite concluir que en general, al aplicar el método de asignación del tipo de suelo sugerido en este estudio, se obtuvieron mejores resultados (estaciones mejor calificadas) para los casos de Nicaragua y Costa Rica que para El Salvador, que es el que aporta la mayor cantidad de registros acelerográficos recopilados.

Finalmente, respecto a las comparaciones entre los niveles de amplificación obtenidos a partir de los promedios de las estaciones con mejor calificación, se observó que las amplificaciones obtenidas en Japón son mayores y las curvas más puntiagudas que para el caso de
América Central, para los cuatro tipos de suelo considerados y para casi todo el rango de periodos, situación que es más evidente en suelos S I (roca) y S III (medio).

Estos resultados permiten iniciar un proceso de clasificación sistemático y homogéneo de las estaciones acelerográficas en América Central, que superan las 200 en la actualidad. Además, se han logrado identificar sitios en los que resulta verdaderamente necesario realizar pruebas más precisas como las geotécnicas, debido a que no fue posible obtener resultados concluyentes utilizando la presente metodología.

\section{AGRADECIMIENTOS}

Esta investigación ha sido posible gracias a la contribución de las siguientes instituciones: Universidad de Costa Rica, Instituto Geológico de Cataluña, Ministerio de Ciencia y Tecnología de Costa Rica y CONOCIT de Costa Rica. Los acelerogramas fueron suministrados por: INETER de Nicaragua, SNET y UCA de El Salvador y LIS de Costa Rica. Gracias al personal del LIS por la ayuda brindada en distintas etapas de esta investigación.

\section{REFERENCIAS}

BSSC, BUILDING SEISMIC SOCIETY COUNCIL, 2003: THE 2003 NEHRP Recommended Provisions for New Buildings and Other Structures, Part 1 (Provisions) and Part II (Commentary).- FEMA 368/369 (2003 ed.).Washington D.C.

BUNDSCHUH, J \& ALVARADO, G. (ed.): Central America: Geology, Resources and Hazards (vol. 1 y 2).- 1311 págs. Taylor \& Francis. The Netherlands. 
DENYER, P. \& ALVARADO, G., 2007: Mapa Geológico de Costa Rica.- Escala 1:400 000, Ed. Librería Francesa, San José.

CENTRO NACIONAL DE REGISTROS, INSTITUTO GEOGRÁFICO NACIONAL, MINISTERIO DE ECONOMÍA DE EL SALVADOR, 2000: Atlas de El Salvador. Cuarta edición.- Escala 1:500 000, Imprenta Scanner color SA de CV, El Salvador.

INETER \& BRG, 2004: Mapa geológico minero de la República de Nicaragua.-
Escala: 1:750 000 Fuente de datos 1995, Managua.

GSJ [GEOLOGICAL SURVEY OF JAPAN], 2010: http://www.gsj.jp/geomap/J-geology/J-geologyE.html. [Consulta: 30/10/ 2010].

ZHAO, JOHN X., IRIKURA, K., ZHANG, J., FUKOSHIMA, Y., SOMERVILLE, P.G., ASANO, A., OHNO, Y., OOUCHI, T., TAKAHASHI, T. \& OGAWA, H. 2006: An empirical site-classification method for strong-motion in Japan using H/V response spectral ratio.- Bull. Sesism. Soc. Am. 96(3): 914-925. 